\title{
Effects of neoadjuvant chemotherapy on the contralateral non-tumor-bearing breast assessed by diffuse optical tomography
}

\author{
Mirella L. Altoe ${ }^{1}$ (D) Kevin Kalinsky², Alessandro Marone ${ }^{1}$, Hyun K. Kim¹ , Hua Guo ${ }^{3}$, Hanina Hibshoosh ${ }^{3}$, \\ Mariella Tejada ${ }^{2}$, Katherine D. Crew ${ }^{2,4}$, Melissa K. Accordino ${ }^{2}$, Meghna S. Trivedi², Dawn L. Hershman ${ }^{2,4}$ and \\ Andreas H. Hielscher ${ }^{1 *}$
}

\begin{abstract}
Background: The purpose of this study is to evaluate whether the changes in optically derived parameters acquired with a diffuse optical tomography breast imager system (DOTBIS) in the contralateral non-tumor-bearing breast in patients administered neoadjuvant chemotherapy (NAC) for breast cancer are associated with pathologic complete response ( $p C R)$.

Methods: In this retrospective evaluation of 105 patients with stage II-III breast cancer, oxy-hemoglobin $\left(\mathrm{ctO}_{2} \mathrm{Hb}\right)$ from the contralateral non-tumor-bearing breast was collected and analyzed at different time points during NAC. The earliest monitoring imaging time point was after 2-3 weeks receiving taxane. Longitudinal data were analyzed using linear mixed-effects modeling to evaluate the contralateral breast $\mathrm{ctO}_{2} \mathrm{Hb}$ changes across chemotherapy when corrected for pCR status, age, and BMI.

Results: Patients who achieved pCR to NAC had an overall decrease of $3.88 \mu \mathrm{M}$ for $\mathrm{ctO}_{2} \mathrm{Hb}(95 \% \mathrm{Cl}, 1.39$ to $6.37 \mu \mathrm{M}), p=.004$, after $2-3$ weeks. On the other hand, non-pCR subjects had a non-significant mean reduction of $0.14 \mu \mathrm{M}(95 \% \mathrm{Cl},-1.30$ to $1.58 \mu \mathrm{M}), p>.05$. Mixed-effect model results indicated a statistically significant negative relationship of $\mathrm{CtO}_{2} \mathrm{Hb}$ levels with $\mathrm{BMl}$ and age.
\end{abstract}

Conclusions: This study demonstrates that the contralateral normal breast tissue assessed by DOTBIS is modifiable after NAC, with changes associated with $\mathrm{pCR}$ after only $2-3$ weeks of chemotherapy.

Keywords: Diffuse optical tomography, Breast cancer, Contralateral breast, Menopausal status, NAC, pCR

\section{Background}

Neoadjuvant chemotherapy (NAC) has become a wellestablished therapy in treating patients with locally advanced breast cancer and consists of 3-6 months of systemic, preoperative treatment. Chemotherapy can modify the density and composition of normal breast tissue. Standardized response evaluation after NAC can be

\footnotetext{
*Correspondence: ahh4614@nyu.edu

'Departments of Biomedical Engineering, New York University Tandon School of Engineering, Brooklyn, NY 11201, USA

Full list of author information is available at the end of the article
}

assessed by residual cancer burden (RCB) index, which is quantified based on primary tumor area, percentage of the tumor area that is invasive cancer, and extent of lymph node involvement. A score of 0 (RCB0) equates to a pathological complete response (pCR) to NAC, and it is defined as the complete disappearance of the tumor in the breast and lymph node (ypT0/is ypNo Mx) at the time of surgery. Non-pCR tumors can be categorized into one of the following three classes: RCB-I (minimal burden), RCB-II (moderate burden), and RCB-III (extensive burden). RCB after NAC is associated with 
improved clinical outcomes, such as disease-free survival, and can predict disease recurrence across different breast cancer subtypes [1-3].

Relatively inexpensive, simple to operate, and welltolerated by patients, diffuse optical tomography (DOT) has shown promising applications in breast cancer detection and monitoring NAC [4-8]. However, the measurement of optically derived parameters in the affected breast is strongly dependent on the tumor location, and DOT suffers from low spatial resolution due to its nonlinear, ill-conditioned, and ill-posed inverse problem [9]. Using a priori information provided by an additional high-resolution anatomical imaging modality, such as magnetic resonance or X-ray, could improve spatial resolution but also nullify the low-cost benefit associated with DOT techniques [10]. Therefore, DOT quantitative measure from the contralateral breast could offer a nontumor dependent measurement to monitor NAC induced changes in the breast tissue and assess treatment outcome. Evaluation of optically derived parameters variation in the contralateral non-tumor-bearing breast following NAC has been previously analyzed [11]. Still, no extensive analyses have been performed to evaluate whether these changes are associated with tumor response in the affected breast. A study published in 2005 [12] reported that chemotherapy could decrease contralateral breast cancer risk in younger women [13]. However, assessment of chemotherapy-related benefits for developing a newly detected breast cancer is still a challenge due to the absence of accurate quantitative metrics.

Over the last decade, our group has developed a diffuse optical tomography breast imaging system (DOTBIS) for a full 3-dimensional tomographic assessment of the entire breast volume and simultaneous bilateral breast imaging. DOTBIS data acquisition is performed without contrast agents or compression and can measure tissue concentration of oxy-hemoglobin $\left(\mathrm{ctO}_{2} \mathrm{Hb}\right)$, deoxy-hemoglobin $(c t H H b)$, and water. Our previous studies have described the utility of DOTBIS in monitoring tumor response to NAC $[7,14]$ and tumor detection [15]. As our study was performed bilaterally on breast cancer patients, DOTBIS quantitative measurements from the contralateral non-tumor-bearing breast were acquired and reported in this paper.

The purpose of this study is to investigate the change of quantitative measurements of functional tissue components, such as hemoglobin concentration and water percentage, measured with DOTBIS in the contralateral non-tumor-bearing breast of patients receiving NAC. We hypothesize that hemoglobin and water values in the unaffected breast, directly related to tissue metabolism and vascular characteristics, are associated with pCR to NAC.

\section{Methods}

Between 2011 and 2019, 105 patients diagnosed with stage II-III breast cancer and scheduled to undergo NAC were consented to participate in a DOTBIS longitudinal research. Menopausal status, body mass index (BMI), and age were retrieved from the patient's electronic medical record. $\mathrm{pCR}$ in the breast was defined as the absence of invasive carcinoma (ypT0/is ypNO Mx) at the time of surgery. Residual Center Burden (RCB) was assessed per established guidelines by Symmans et al. [16]. This study was approved by the institutional review board at Columbia University Irving Medical Center (CUIMC).

\section{DOTBIS imaging acquisition}

Measurements were performed by a continuous wave dynamic imaging system (i.e., DOTBIS) that provides full three-dimensional tomographic images of both breasts simultaneously without requiring the use of contrast agents or compression. A more detailed description of the system can be found in previous reports $[15,17]$. Total measurement time varied between 6 and $8 \mathrm{~min}$ in addition to $5 \mathrm{~min}$ required to set up the machine. Nearinfrared light transmission was carried out using four laser diodes operating at $765,808,827$, and $905 \mathrm{~nm}$. The patient interface consists of two sets of four rings that use up to 32 sources and 64 detectors, depending on the patient's breast cup size, for optical data collection. The spatial distribution of chromophore concentration of $c t \mathrm{O}_{2} \mathrm{Hb}, \mathrm{ctHHb}$, and water was obtained by a transporttheory-based PDE-constrained multispectral model [18], which uses the diffusion approximation of the equation of radiative transfer to describe the light propagation in such scattering-dominated media as the breast tissue. Additional features such as $c t \mathrm{THb}$ and $\mathrm{StO}_{2} \%$ were defined respectively as Eq. (1) and Eq. (2). For visualization, maximum intensities projections (MIP) $[19,20]$ were obtained from the reconstructed 3D chromophore concentration maps. MIP projects the acquired data volume into a view plane, which can be obtained by finding the voxels with maximum intensity along a chosen axis, Fig. 1.

$$
\begin{aligned}
& c t \mathrm{THb}=c t \mathrm{O}_{2} \mathrm{Hb}+c t H H b \\
& \mathrm{StO}_{2} \%=\frac{c t \mathrm{O}_{2} \mathrm{Hb}}{c t \mathrm{THb}} \times 100 \%
\end{aligned}
$$

\section{NAC imaging protocol}

Sixty-nine patients received 12 cycles of weekly paclitaxel (with or without the addition of carboplatin) followed by four cycles of doxorubicin and cyclophosphamide given every 2 weeks with growth factor support $(\mathrm{T} \times 12 / \mathrm{AC} \times$ 


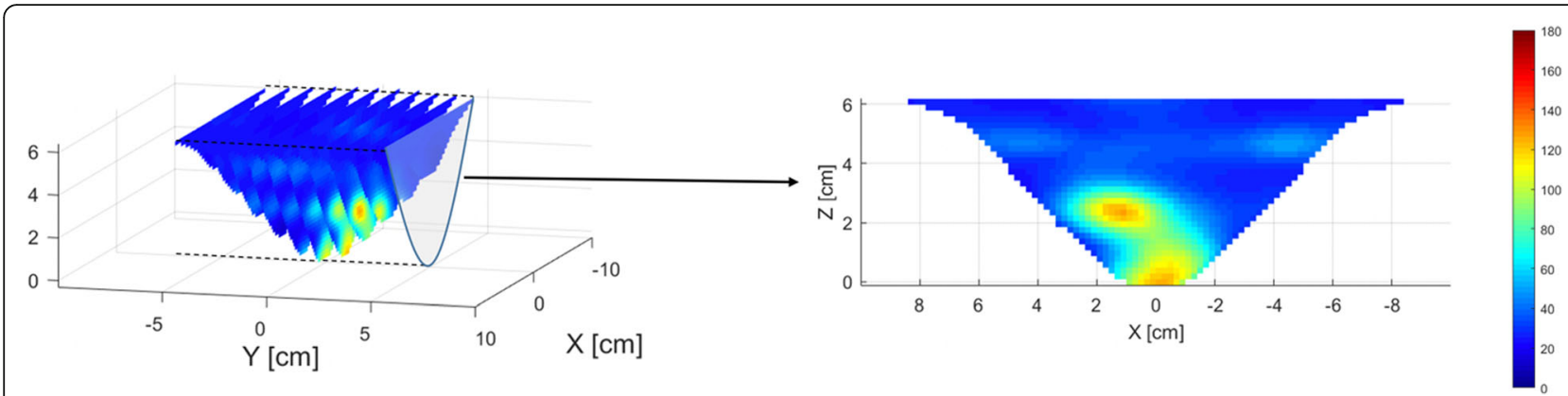

Fig. 1 Visual scheme of how maximum intensity projection (MIP) images are generated from the DOTBIS acquired ctTHb 3D map ( $\mu M$ ). MIP is obtained by finding the maximum intensity for each voxel along the $y$-axis direction. The maximum voxel values are registered into a single view plane for visualization purposes only

4 or $\mathrm{T} / \mathrm{C} \times 12 / \mathrm{AC} \times 4$ ), and DOTBIS bilateral measurement was acquired at six different time points: before starting NAC regimen (TP0), after 2 weeks of taxane infusions (TP1), after 4 weeks of taxane (TP2), at the end of the taxane regimen and before starting $\mathrm{AC}$ cycles (TP3), after two cycles of AC (TP4), and at the end of NAC and before surgery (TP5). Thirty-six patients received other taxane-based NAC regimens without an anthracycline, and bilateral imaging was performed only three times (baseline (TP0), 2-3 weeks after the first taxane infusion (TP1), and at the end of NAC and before surgery (TP5)), Fig. 2.

\section{Statistical analysis}

An independent-samples $t$ test was run to determine if there were differences in baseline DOTBIS parameters between pre-and postmenopausal subjects and pCR status (pCR and non-pCR patients). Feature importance was calculated by using Random Forest [21] modelspecific metrics from the $\mathrm{R}$ package "caret" [22]. The
Random Forest algorithm has a built-in feature selection, mean decrease Gini (Gini importance) [23], which can be used as a score that provides a relative ranking of feature relevance. A paired-samples $t$ test was used to determine whether there was a statistically significant mean difference in $\mathrm{ctO}_{2} \mathrm{Hb}$ levels measured at baseline and TP1 between pCR and non-pCR and between both menopausal groups. To account for repeated measures, a linear mixed-effect model was designed to evaluate if there is a difference in the average longitudinal evolution of $c t \mathrm{O}_{2} \mathrm{Hb}$ when we correct for pCR status (or age and $\mathrm{BMI}$ ). Significance was assumed at a confidential interval of $95 \%(\alpha=0.05)$.

\section{Result}

\section{Patient summary}

From a total of 105 subjects, 18 were excluded from the final analysis data set. The main reasons for exclusion were as follows: patient withdrew from the study $(n=4)$, baseline DOTBIS measurement not collected due to

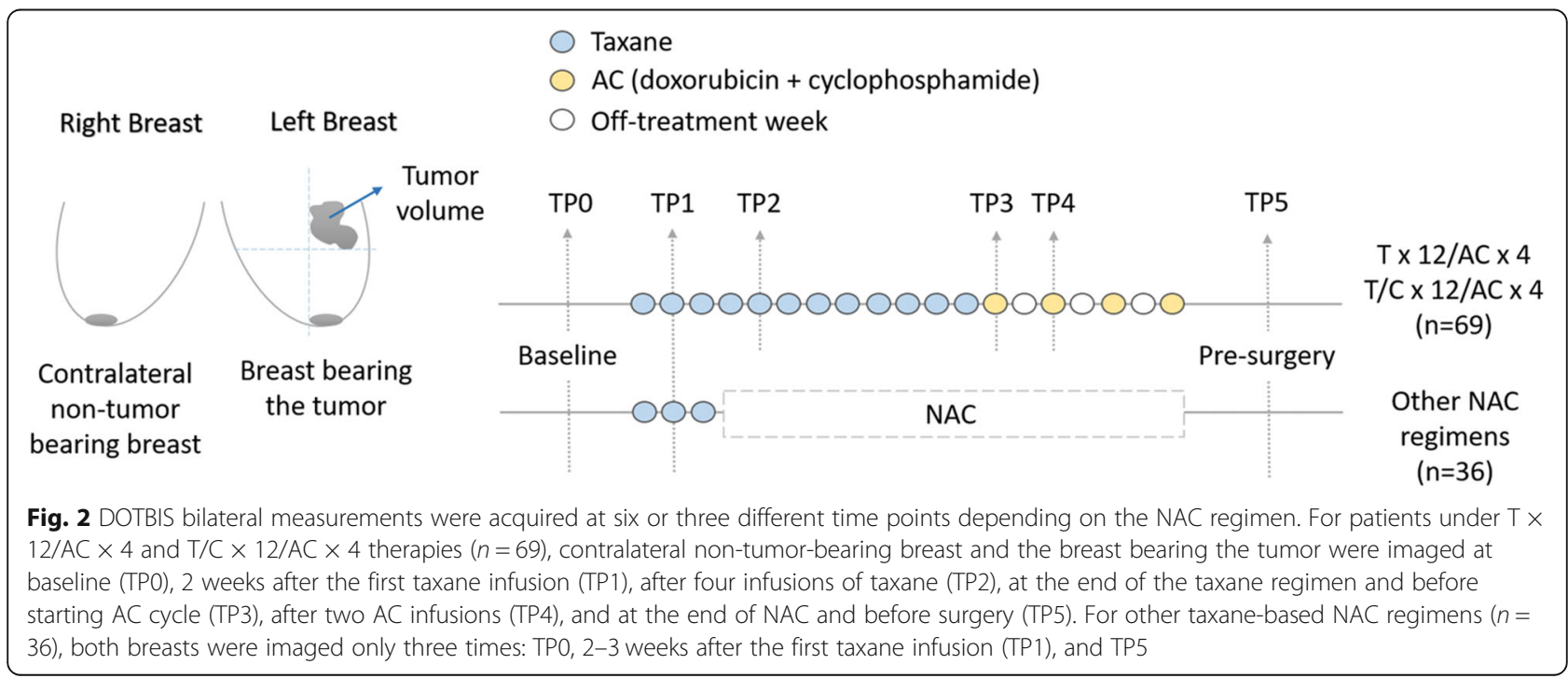


instrumentation failure $(n=8)$, patient receiving non-FDA approved drug regimen $(n=1)$, bilateral breast cancer $(n=1)$, and machine operator error $(n=4)$. Not all the patients had their DOTBIS reconstruction available for all of their imaging time points due to patient declining imaging at that particular DOTBIS time point or machine malfunction. Overall, 81 (93.1\%) patients had DOTBIS images successfully reconstructed at baseline (TP0), 69 (79.3\%) patients at 2-3 weeks from taxane initiation (TP1), and 52 $(59.8 \%)$ at the pre-surgical time point (TP5). Missing TP5 data points were due to patient declined to be imaged (18/ 35), machine operator did not collect/save the data correctly (5/35), and data not collected due to machine under repair $(12 / 35)$. Out of the 58 patients who were administered the $\mathrm{T} \times 12 / \mathrm{AC} \times 4(n=54)$ regimen or $\mathrm{T} / \mathrm{C} \times 12 / \mathrm{AC} \times 4(n=4), 46(79 \%)$ had their data acquired and reconstructed at TP2, $43(74 \%)$ at TP3, and $40(69 \%)$ at TP4. Non-anthracycline regimens accounted for the remaining 29 patients. Patient and clinical characteristics are summarized in Table 1.

\section{Baseline DOTBIS-measured features}

Contralateral $c t T H b, c t \mathrm{O}_{2} \mathrm{Hb}, c t \mathrm{HHb}$, water, and $\mathrm{StO}_{2}$ values were available for all the 87 patients considered for analysis at different time points across NAC. At the baseline level, there was no statistical difference in any of the DOTBIS-measured features between pCR and non-pCR patients $(p>0.05)$. Fifty-two patients $(59.8 \%)$ were premenopausal women, 34 (39.1\%) were postmenopausal, and for one patient (1.1\%), her menopausal status was uncertain. An independent samples $t$ test was run to determine if there were differences in baseline DOTBIS parameters between pre-and postmenopausal subjects. Pre-menopausal women had higher mean $c t T H b(33.30 \pm 12.22 \mu \mathrm{M})$ than post-menopausal women $(24.92 \pm 7.78 \mu \mathrm{M})$, a statistically significant difference of $8.39 \mu \mathrm{M}(95 \% \mathrm{CI}, 3.96$ to 12.81$), \mathrm{t}(79)=$ 3.772, $p<.0005$. ct $\mathrm{O}_{2} \mathrm{Hb}$ levels were also higher for premenopausal women $(19.36 \pm 8.51 \mu \mathrm{M})$ than postmenopausal $(13.24 \pm 4.89 \mu \mathrm{M})$. The difference in means for water was also statistically significant between pre$(48.37 \pm 9.33 \%)$ and postmenopausal $(40.48 \pm 12.14 \%)$ patients, $p=.001$.

\section{Contralateral breast DOTBIS feature selection}

To avoid severe multicollinearity or overfitting, variable importance with random forest was performed to find the most relevant $\mathrm{pCR}$ status classification feature.

Table 1 Patient and clinical characteristics for all the 87 patients considered for analysis

\begin{tabular}{|c|c|c|c|}
\hline Patient and clinical characteristics & Number of patients & Age $($ mean $\pm S D)$ & $\mathrm{BMI}($ mean $\pm \mathrm{SD})$ \\
\hline \multicolumn{4}{|l|}{ Chemotherapy and targeted therapies } \\
\hline $\mathrm{T} \times 12 / \mathrm{AC} \times 4$ & 54 & $48.48 \pm 11.52$ & $29.89 \pm 7.18$ \\
\hline $\mathrm{TCHP} \times 6$ & 21 & $53.80 \pm 10.04$ & $29.307 \pm 9.49$ \\
\hline TC $\times 6$ & 6 & $59.67 \pm 12.24$ & $30.54 \pm 3.24$ \\
\hline $\mathrm{T} / \mathrm{C} \times 12 / \mathrm{AC} \times 4$ & 4 & $39.25 \pm 8.65$ & $30.05 \pm 2.29$ \\
\hline $\mathrm{TC} \times 4$ & 1 & 30 & 18.11 \\
\hline $\mathrm{THP} \times 12 / \mathrm{AC} \times 4$ & 1 & 40 & 32.23 \\
\hline \multicolumn{4}{|l|}{ Menopausal status } \\
\hline Premenopausal & 52 & $41.65 \pm 6.90$ & $29.48 \pm 7.91$ \\
\hline Postmenopausal & 34 & $61.63 \pm 6.00$ & $30.10 \pm 6.82$ \\
\hline Uncertain & 1 & 56 & 27.16 \\
\hline Tumor characteristics & Number of tumors & & \\
\hline \multicolumn{4}{|l|}{ Treatment response classification } \\
\hline \multicolumn{4}{|l|}{$\mathrm{pCR}$} \\
\hline RCB 0 & 36 & & \\
\hline \multicolumn{4}{|l|}{ Non-pCR } \\
\hline RCB I & 10 & & \\
\hline RCB ॥ & 27 & & \\
\hline RCB III & 11 & & \\
\hline RCB score not available & 3 & & \\
\hline
\end{tabular}

Abbreviations and acronyms: $T \times 12 / \mathrm{AC} \times 4,12$ cycles of weekly paclitaxel followed by 4 cycles of doxorubicin and cyclophosphamide given every 2 weeks with growth factor support; T/C $\times 12 / A C \times 4,12$ cycles of weekly paclitaxel followed by 4 cycles of doxorubicin and cyclophosphamide given every 2 weeks with growth-factor support with an addition of carboplatin to paclitaxel; TC $\times 6$, six cycles Cytoxan plus Taxotere; TC $\times 4$, four cycles Cytoxan plus Taxotere; TCHP, six cycles of docetaxel, carboplatin, and trastuzumab plus pertuzumab; THP/AC, paclitaxel, trastuzumab, and pertuzumab followed by doxorubicin and cyclophosphamide; pCR, pathologic complete response; RCB, residual cancer burden; BMI, body mass index; SD, standard deviation 
Looking at the changes of all the DOTBIS-measured features after 2-3 weeks under $\mathrm{NAC}, \mathrm{ctO}_{2} \mathrm{Hb}$ was selected as the best predictor characterized by the highest mean decrease impurity (Gini importance). For this feature, lower values are associated with poor response to NAC (non-pCR).

\section{$\mathrm{ctO}_{2} \mathrm{Hb}$ changes in the contralateral breast after 2-3 weeks under NAC and pCR status}

Thirty-seven subjects (42.5\%) achieved pCR and 50 $(57.5 \%)$ were classified as non-pCR. A paired $t$ test was used to determine whether there was a statistically significant mean difference between baseline ${ }^{2} \mathrm{O}_{2} \mathrm{Hb}$ and after 2-3 weeks under NAC (TP1). Overall, NAC elicited a mean decrease of $1.57 \mu \mathrm{M}$ for $c t \mathrm{O}_{2} \mathrm{Hb}$ levels $(95 \% \mathrm{CI}$, 0.28 to $3.32 \mu \mathrm{M}), \mathrm{t}(67)=2.43, p=.022$, in the contralateral non-tumor-bearing breast. Looking at the pCR group, NAC elicited an overall decrease of $3.88 \mu \mathrm{M}$ for $c t \mathrm{O}_{2} \mathrm{Hb}(95 \% \mathrm{CI}, 1.39$ to $6.37 \mu \mathrm{M}), p=.004$. On the other hand, the non-pCR subjects had a non-significant mean reduction of $0.14 \mu \mathrm{M}$ for $c t \mathrm{O}_{2} \mathrm{Hb}(95 \% \mathrm{CI},-1.30$ to $1.58 \mu \mathrm{M}), p=.845$, Fig. 3 .

$\mathrm{CtO}_{2} \mathrm{Hb}$ longitudinal analysis in the contralateral breast during NAC: PCR status, age, and BMI as model predictors For the longitudinal multilevel mixed-effects analysis, ct $\mathrm{O}_{2} \mathrm{Hb}$ from all patients $(\mathrm{n}=87)$ was analyzed at threetime imaging points, TP0, TP1, and TP5, which are the imaging time points in common to all the patients regardless of their chemotherapy regimen. We fit a linear mixed-effects model with $c t \mathrm{O}_{2} \mathrm{Hb}$ as the outcome variable, with fixed effects of pCR status, BMI and age, and time as a random effect (and fixed effect) and random slope across individuals. We were also particularly interested in the group-occasion interaction between time and $\mathrm{pCR}$ status, as this reflects a potential difference in time evolution between the pCR status groups. There was a significant interaction between $c t \mathrm{O}_{2} \mathrm{Hb}$ over time and $\mathrm{pCR}$ status. $\mathrm{pCR}$ patients had a significant reduction in $\mathrm{ctO}_{2} \mathrm{Hb}$ levels at TP1 $(3.53 \mu \mathrm{M}, p=.0053)$ than nonpCR, but not statistically significant at TP5 $(2.59 \mu \mathrm{M}$, $p=.131)$. This trend can also be observed in the iteration plot of $\mathrm{ctO}_{2} \mathrm{Hb}$ levels over the different time points for both $\mathrm{pCR}$ and non-pCR groups, Fig. 4. Significant main effects of age $(p=.0015)$ and BMI $(p=.0011)$ were also found, Table 2, characterized by a negative relationship, older and overweighted patients had lower $c t \mathrm{O}_{2} \mathrm{Hb}$ levels. Figure 5 (pCR) and Fig. 6 (non-pCR) illustrate the hemoglobin map for two premenopausal patients imaged at three different time points during NAC regimen.

\section{Discussion}

We investigated whether the changes of DOTBIS features in the contralateral healthy breast are associated with therapy outcome in patients with stage II-III breast cancer receiving NAC. We observed that hemoglobin level is modifiable throughout the course of NAC. The magnitude of change is associated with $\mathrm{pCR}$ after only 2-3 weeks with the initiation of a taxane-based regimen. Patients who achieved a pCR to NAC had a more significant reduction in DOTBIS-measured features than the non-pCR group.

Previous studies have demonstrated statistically significant changes in optically derived parameters in breast cancer patients undergoing NAC and their correlation with tumor response [5, 14, 24-28]. However, most of these studies looked at the tissue composition of the tumor volume, and few of them only used the contralateral healthy breast data as a normalizing factor for the total tissue hemoglobin concentration within the tumor region. The measurement of optically derived parameters in the affected breast is strongly dependent on selecting the tumor location or region of interest (ROI). Yet, it is well known that breast optical imaging has limitations regarding spatial resolution. Therefore, the optically derived parameters collected from the
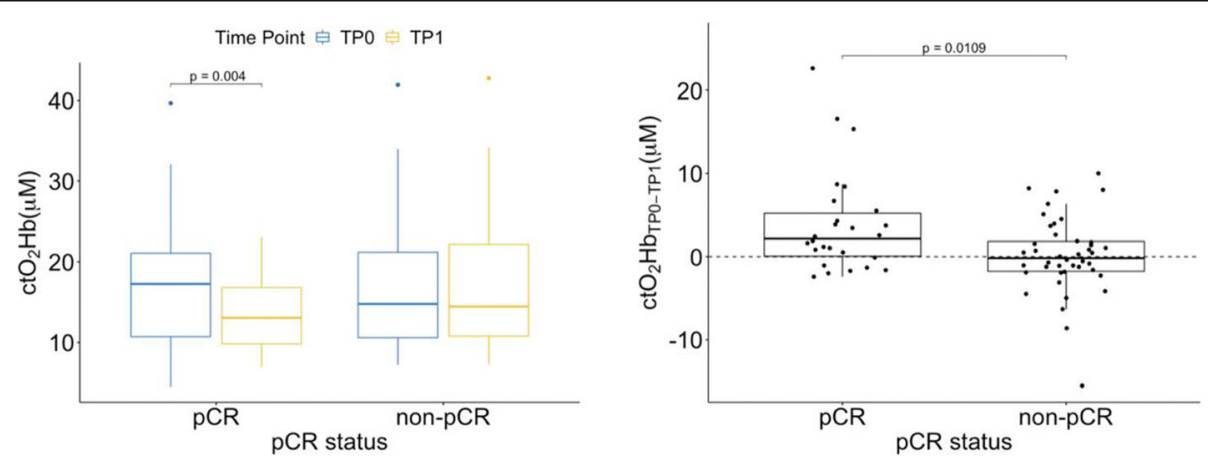

Fig. 3 Left: $\mathrm{CtO}_{2} \mathrm{Hb}$ changes after 2-3 weeks of chemotherapy showing a significant decrease for pCR patients but a non-significant change for the non-pCR group. Right: Box plot of the difference between $\mathrm{ctO}_{2} \mathrm{Hb}$ levels measured at baseline and after 2-3 weeks under Taxane regimen (TP1) for pCR and non-pCR patients showing a significant difference between both groups. $\mathrm{CtO}_{2} \mathrm{Hb}$ reduction levels at TP1 were larger for PCR patients 

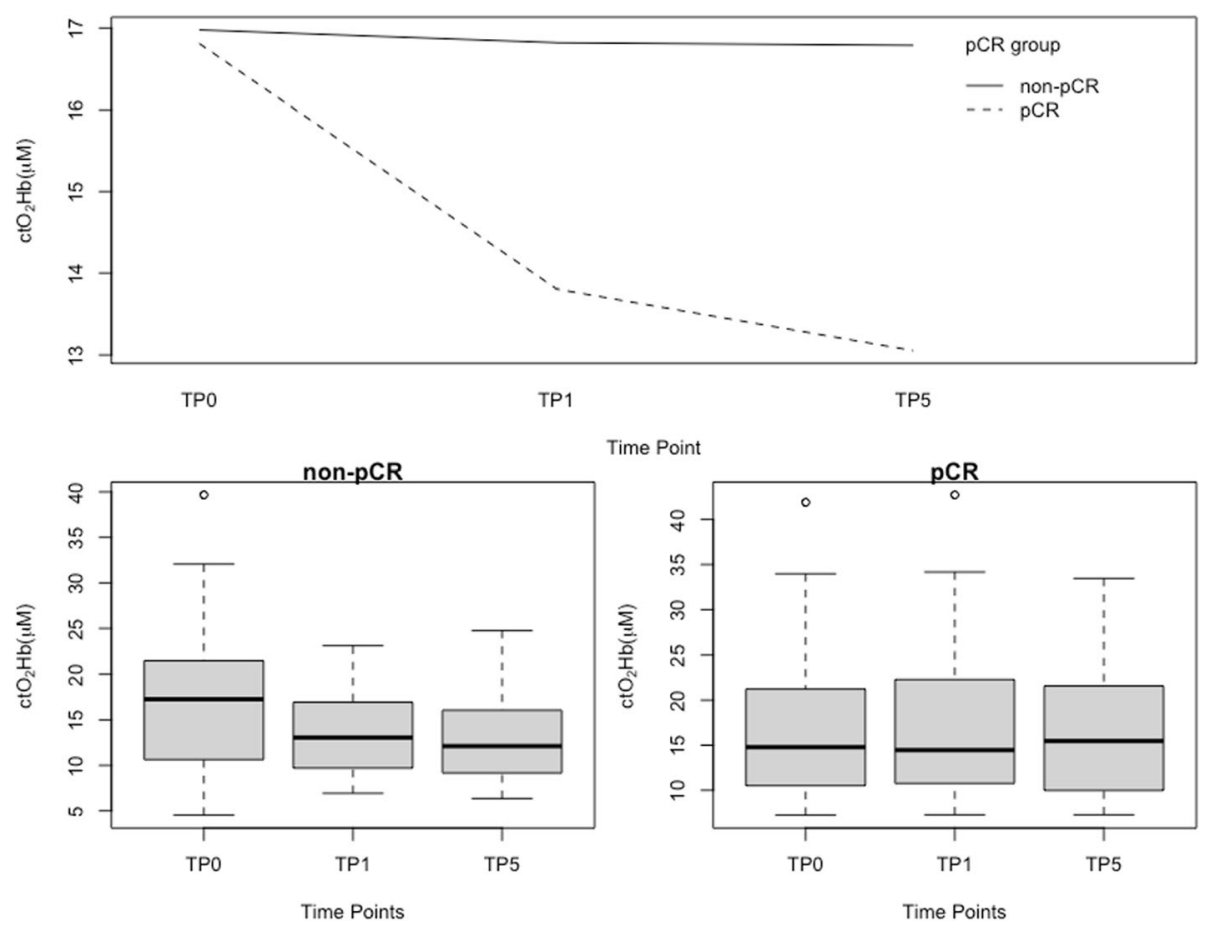

Fig. 4 Top: Plot illustrates the interaction between the mean response for two-way combination of factors: time and pCR status over three-time points. Bottom: Box plots and 95\% confidence intervals for $\mathrm{ctO}_{2} \mathrm{Hb}$ separated by tumor response to NAC: pCR (Left) non-pCR (Right) groups

contralateral unaffected breast can offer a more unbiased and non-ROI dependent measurement to follow up the course of changes in the breast tissue during NAC and associations with treatment outcome. The only two previous studies [11, 29], which evaluated optical imaging changes during NAC in the contralateral normal tissue, did not perform analyses regarding tumor response. By associating contralateral healthy breast data with tumor response, we could provide a potential marker for evaluating chemotherapy regimens' efficacy early in the treatment course.

Table 2 Summary for the longitudinal model analysis: estimated model fixed effects

\begin{tabular}{lllll}
\hline Effect & Estimate & Std. error & $\mathbf{t}$ & $\boldsymbol{p}$ value \\
\hline Intercept & 33.41 & 3.65 & 9.15 & $<0.0005$ \\
pCR status & 0.76 & 1.61 & 0.47 & 0.64 \\
Time TP1 & -3.60 & 0.97 & -3.72 & 0.0003 \\
Time TP5 & -3.73 & 1.32 & -2.82 & 0.0056 \\
BMI & -0.29 & 0.008 & -3.37 & 0.0011 \\
Age & -0.17 & 0.052 & -3.39 & 0.0015 \\
TP1: pCR status & 3.53 & 1.24 & 2.84 & 0.0053 \\
TP5: pCR status & 2.59 & 1.70 & 1.52 & 0.1306 \\
\hline
\end{tabular}

Abbreviations and acronyms: $\mathrm{pCR}$, pathologic complete response; TP1, time point after 2-3 weeks under chemotherapy; TP5, time point after ending chemotherapy
A similar association with $\mathrm{pCR}$ is also reported in the literature for background parenchymal enhancement (BPE) at breast magnetic resonance (MR) imaging in the contralateral normal breast. Several groups [30-33] have demonstrated an association between BPE and tumor response after receiving NAC in the last 4 years. Chen et al. found that BPE showed a significant decrease in those with pCR compared to non-pCR for pre/peri-menopausal patients after starting NAC [30]. Next, Preibsch et al. investigated the influence of chemotherapy on the degree of $\mathrm{BPE}$ and found correlations between tumor response and qualitative assessment of BPE following NAC [31]. You et al. also reported a positive association between overall BPE reduction at the early stage of NAC and tumor response, especially in patients with hormone receptor-negative breast cancers [32].

Given enough evidence that optical imaging is a powerful tool to study the breast tissue's structural and vascular properties [34], $c t T H b$ and $c t \mathrm{O}_{2} \mathrm{Hb}$ are thought to be correlated to the amount of blood flow in the fibroglandular tissue and represent breast vascular supply and perfusion. The significant large reduction of DOTBIS-measured features for the premenopausal group may be explained by the ovarian function suppression that affects estrogen secretion and leads to a secondary change in breast tissue composition and its vascular characteristics $[35,36]$. A review published in 


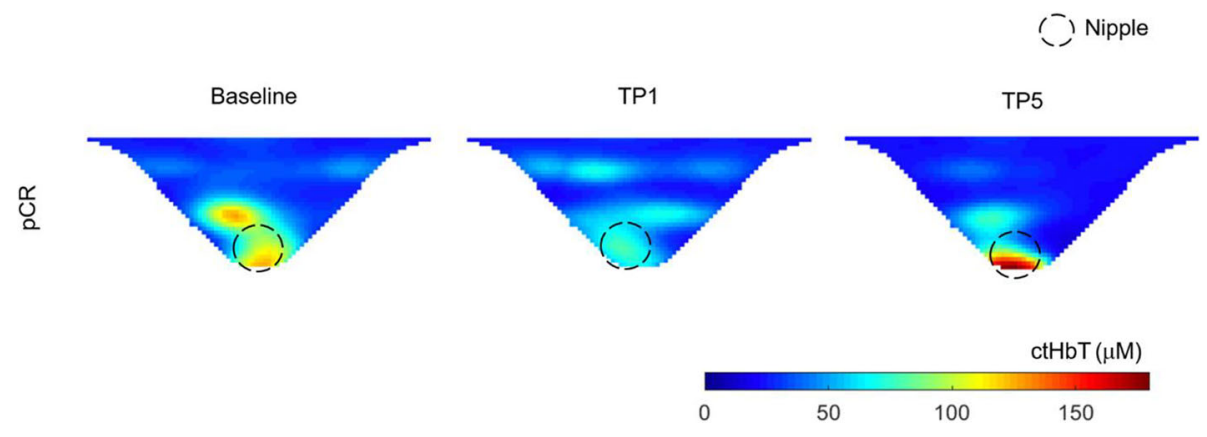

Fig. 5 Corresponding hemoglobin levels map for the contralateral unaffected breast of 47-year-old women who achieved pCR after TCHP therapy for HER2 + breast cancer. MIP images (axial orientation) were acquired at baseline, after 2-3 weeks after NAC initiation (TP1), and at the pre-surgical time point (TP5)

2005 [12] also concluded that younger women had much larger benefits from chemotherapy than older patients, potentially due to the ovarian ablation induced by chemotherapeutic agents. Fibroglandular tissue (FGT) and BPE-MRI were also reported as markedly influenced by menopausal status, significantly decreasing after menopause for most women included in the study [37]. On the other hand, substantial changes in DOTBISmeasured parameters for those with $\mathrm{PCR}$ regardless of their menopausal status could also be associated with the direct damage of the vascular morphology and density in normal tissue caused by chemotherapy, as investigated by Miller et al. [38].

Besides, the mixed-effect model results indicated a statistically significant negative relationship between $c t \mathrm{O}_{2} \mathrm{Hb}$ and BMI. DOTBIS-measured $\mathrm{ctO}_{2} \mathrm{Hb}$ is thought to be correlated to breast density since it is related to the arterial blood supply carrying oxygen to meet the fibroglandular tissue metabolic demand [29]. In contrast, BMI is inversely related to percent density and acts as a co-founder of each other's effects [39-42]. In other words, overweight is correlated to a greater amount of fat in the breast, reflecting a larger non-dense tissue area in the mammogram and, consequently, lower percent density classification [41].

A limitation of our study was we did not reliably capture the patient's menstrual period during treatment and around the time of scans. Besides that, since the majority of the patients in our study did not have breast MR imaging before and after NAC treatment, we were not able to expand our analyses to correlations between MR based quantitative measurements and DOTBIS features. Finally, a more extensive study is also required to identify potential optical predictors of long-term outcomes in breast cancer patients who undergo NAC and understand chemotherapy's effect on reducing contralateral cancer risk.

\section{Conclusion}

In summary, this is the first study to confirm that optical derived metrics, measured by DOTBIS, in the contralateral non-tumor-bearing breast tissue, are modifiable with NAC treatment. $c t \mathrm{O}_{2} \mathrm{Hb}$ changes are proportional to the degree of pCR after 2-3 weeks of taxane-based regimen.

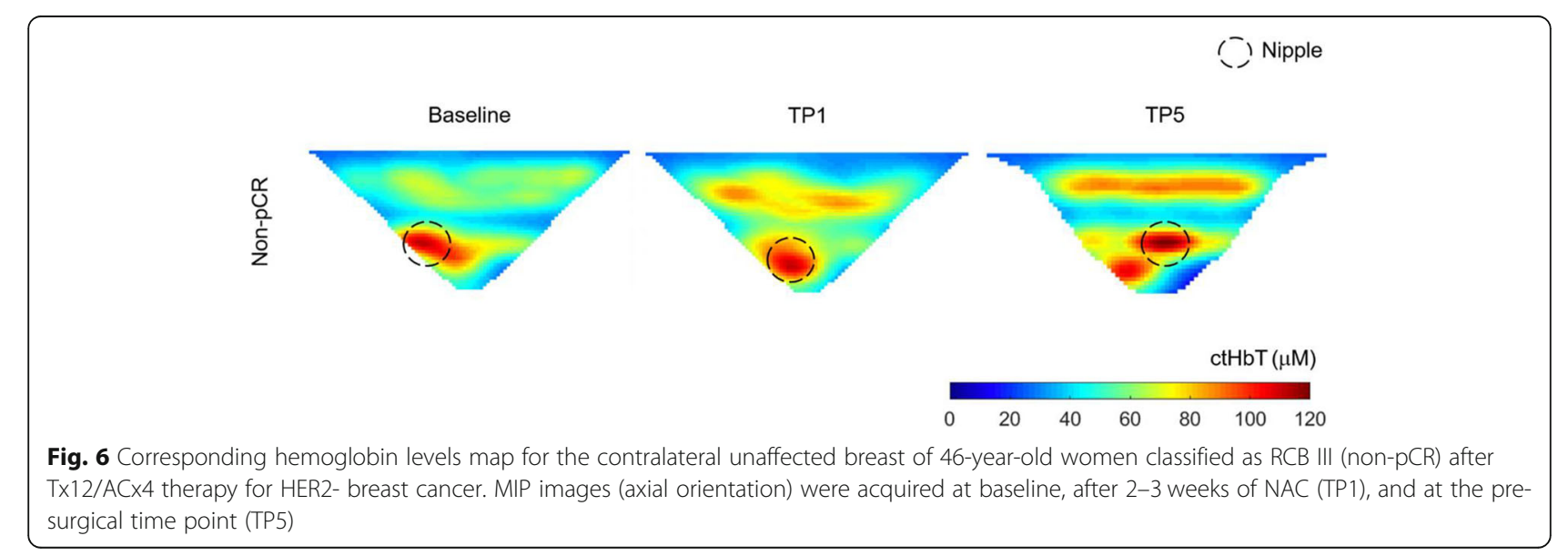




\section{Acknowledgements}

Not applicable.

\section{Authors' contributions}

Provision of study materials or patients was made by KK, KDC, MKA, MST, and DLH. MLA and MT were responsible for data collection and imaging MLA, AM, and HKK were responsible for data processing and image analysis. MLA, KK, DLH, and AHH were responsible for data analysis and interpretation. $\mathrm{HG}$ and $\mathrm{HH}$ analyzed the pathological clinical samples. MLA, KK, DLH, and $\mathrm{AHH}$ wrote the manuscript with assistance from the other authors. All authors read and approved the final manuscript.

\section{Funding}

This work was supported in part by a grant from the Columbia Biomedical Engineering Technology Accelerator (BiomedX) and the Breast Cancer Research Foundation. Kevin Kalinsky was supported in part by the Irving Scholar Award. Furthermore, Mirella L. Altoé was supported in part by a fellowship from CNPq/LASPAU - Brazil [207913/2014-5], and Alessandro Marone was supported in part by a Personalized Medicine Fellowship of the Irving Institute for Clinical and Translational Research at Columbia University in the City of New York.

\section{Availability of data and materials}

The datasets used and analyzed during the current study are available from the corresponding author on reasonable request.

\section{Ethics approval and consent to participate}

All subjects provided informed written consent and participated in this study under a clinical protocol approved by the Institutional Review Board at the Columbia University Irving Medical Center.

\section{Consent for publication}

Not applicable.

\section{Competing interests}

The authors declare that they have no competing interests.

\section{Author details}

'Departments of Biomedical Engineering, New York University Tandon School of Engineering, Brooklyn, NY 11201, USA. ²Department of Medicine, Division of Hematology/Oncology, Columbia University Irving Medical Center, New York, NY 10032, USA. ${ }^{3}$ Department of Pathology and Cell Biology, Columbia University Irving Medical Center, New York, NY 10032, USA. ${ }^{4}$ Department of Epidemiology, Columbia University Irving Medical Center, New York, NY 10032, USA.

\section{Received: 5 May 2020 Accepted: 20 January 202}

\section{Published online: 31 January 2021}

\section{References}

1. Cortazar $\mathrm{P}$, et al. Pathological complete response and long-term clinical benefit in breast cancer: the CTNeoBC pooled analysis. Lancet. 2014; 384(9938):164-72. https://doi.org/10.1016/50140-6736(13)62422-8.

2. Bonadonna G, Valagussa P, Brambilla C, Ferrari L. Preoperative chemotherapy in operable breast cancer. Lancet. 1993;341(8858):1485. https://doi.org/10.1016/0140-6736(93)90933-8.

3. Cameron DA, et al. Primary systemic therapy for operable breast cancer 10-year survival data after chemotherapy and hormone therapy. Br. J. Cancer. 1997;76(8):1099. https://doi.org/10.1038/bjc.1997.514.

4. B. J. Tromberg, B. W. Pogue, K. D. Paulsen, A. G. Yodh, D. A. Boas, and A. E. Cerussi, "Assessing the future of diffuse optical imaging technologies for breast cancer management," Med. Phys., 35, no. 6Part1, pp. 2443-2451, 2008, doi: https://doi.org/10.1118/1.2919078.

5. Cerussi A, et al. Predicting response to breast cancer neoadjuvant chemotherapy using diffuse optical spectroscopy. Proc Natl Acad Sci U S A. 2007:104(10):4014-9. https://doi.org/10.1073/pnas.0611058104.

6. Cong $W$, Intes $X$, Wang G. Optical tomographic imaging for breast cancer detection. J Biomed Opt. 2017:22(9):1-6. https://doi.org/10.1117/1.JBO.22.9 096011
7. Gunther JE, et al. Dynamic diffuse optical tomography for monitoring neoadjuvant chemotherapy in patients with breast cancer. Radiology. 2018; 287(3):778-86. https://doi.org/10.1148/radiol.2018161041.

8. Choe $R$, et al. Differentiation of benign and malignant breast tumors by invivo three-dimensional parallel-plate diffuse optical tomography. J Biomed Opt. 2009;14(2):024020. https://doi.org/10.1117/1.3103325.

9. Chance B, Cooper CE, Delpy DT, Reynolds EOR, Arridge SR, Schweiger M. Image reconstruction in optical tomography. Philos Trans R Soc Lond Ser B Biol Sci. 1997;352(1354):717-26. https://doi.org/10.1098/rstb.1997.0054.

10. Azizi L, Zarychta K, Ettori D, Tinet E, Tualle J-M. Ultimate spatial resolution with diffuse optical tomography. Opt Express. 2009;17(14):12132-44. https:// doi.org/10.1364/OE.17.012132.

11. O'Sullivan TD, et al. Optical imaging correlates with magnetic resonance imaging breast density and reveals composition changes during neoadjuvant chemotherapy. Breast Cancer Res. 2013;15(1):R14. https://doi. org/10.1186/bcr3389.

12. Early Breast Cancer Trialists' Collaborative Group (EBCTCG). Effects of chemotherapy and hormonal therapy for early breast cancer on recurrence and 15-year survival: an overview of the randomised trials. Lancet Lond Engl. 2005;365(9472):1687-717. https://doi.org/10.1016/501406736(05)66544-0.

13. Shahedi K, Emanuelsson M, Wiklund F, Gronberg H. High risk of contralatera breast carcinoma in women with hereditary/familial non-BRCA1/BRCA2 breast carcinoma. Cancer. 2006;106(6):1237-42. https://doi.org/10.1002/cncr. 21753.

14. Lim EA, et al. Diffuse optical tomography changes correlate with residual cancer burden after neoadjuvant chemotherapy in breast cancer patients. Breast Cancer Res Treat. 2017;162(3):533-40. https://doi.org/10.1007/s10549017-4150-7.

15. Flexman ML, et al. Optical biomarkers for breast cancer derived from dynamic diffuse optical tomography. J Biomed Opt. 2013;18(9):096012. https://doi.org/10.1117/1.JBO.18.9.096012

16. Symmans WF, et al. Measurement of residual breast cancer burden to predict survival after neoadjuvant chemotherapy. J Clin Oncol Off J Am Soc Clin Oncol. 2007;25(28):4414-22. https://doi.org/10.1200/JCO.2007.10.6823.

17. Flexman ML, et al. Digital optical tomography system for dynamic breast imaging. J Biomed Opt. 2011;16(7):076014. https://doi.org/10.1117/1. 3599955.

18. Kim HK, Flexman M, Yamashiro DJ, Kandel JJ, Hielscher AH. PDE-constrained multispectral imaging of tissue chromophores with the equation of radiative transfer. Biomed Opt Express. 2010;1(3):812-24. https://doi.org/10. 1364/BOE.1.000812.

19. Cody DD. AAPM/RSNA physics tutorial for residents: topics in CT RadioGraphics. 2002;22(5):1255-68. https://doi.org/10.1148/radiographics.22. 5.g02se041255.

20. Prokop M, Shin HO, Schanz A, Schaefer-Prokop CM. Use of maximum intensity projections in CT angiography: a basic review. RadioGraphics. 1997; 17(2):433-51. https://doi.org/10.1148/radiographics.17.2.9084083.

21. Breiman L. Random forests. Mach Learn. 2001;45(1):5-32. https://doi.org/10. 1023/A:1010933404324.

22. A. Liaw and M. Wiener, "Classification and regression by RandomForest," Forest 23. 2001;Vol.2/3:18-22. https://www.r-project.org/doc/Rnews/ Rnews 2002-3.pdf

23. Chen R-C, Dewi C, Huang S-W, Caraka RE. Selecting critical features for data classification based on machine learning methods. J Big Data. 2020;7(1):52 https://doi.org/10.1186/s40537-020-00327-4.

24. Choe R, Durduran T. Diffuse optical monitoring of the neoadjuvant breast cancer therapy. IEEE J Sel Top Quantum Electron Publ IEEE Lasers ElectroOpt Soc. 2012;18(4):1367-86. https://doi.org/10.1109/JSTQE.2011.2177963.

25. Zhou C, et al. Diffuse optical monitoring of blood flow and oxygenation in human breast cancer during early stages of neoadjuvant chemotherapy. J Biomed Opt. 2007;12(5):051903. https://doi.org/10.1117/1.2798595.

26. Ueda $\mathrm{S}$, et al. Baseline tumor oxygen saturation correlates with a pathologic complete response in breast cancer patients undergoing neoadjuvant chemotherapy. Cancer Res. 2012;72(17):4318-28. https://doi.org/10.1158/ 0008-5472.CAN-12-0056.

27. Jiang $\mathrm{S}$, et al. Evaluation of breast tumor response to neoadjuvant chemotherapy with tomographic diffuse optical spectroscopy: case studies of tumor region-of-interest changes. Radiology. 2009;252(2):551-60. https:// doi.org/10.1148/radiol.2522081202. 
28. Cerussi AE, Tanamai WW, Hsiang D, Butler J, Mehta RS, Tromberg BJ. Diffuse optical spectroscopic imaging correlates with final pathological response in breast cancer neoadjuvant chemotherapy. Philos Transact A Math Phys Eng Sci. 2011;369(1955):4512-30. https://doi.org/10.1098/rsta.2011.0279.

29. Altoe ML, et al. Diffuse optical tomography of the breast: a potential modifiable biomarker of breast cancer risk with neoadjuvant chemotherapy. Biomed Opt Express. 2019;10(8):4305-15. https://doi.org/10.1364/BOE.10. 004305.

30. Chen JH, Yu HJ, Hsu C, Mehta RS, Carpenter PM, Su MY. Background parenchymal enhancement of the contralateral normal breast: association with tumor response in breast cancer patients receiving neoadjuvant chemotherapy. Transl Oncol. 2015;8(3):204-9. https://doi.org/10.1016/j. tranon.2015.04.001.

31. Preibsch $\mathrm{H}$, et al. Background parenchymal enhancement in breast MRI before and after neoadjuvant chemotherapy: correlation with tumour response. Eur Radiol. 2016;26(6):1590-6. https://doi.org/10.1007/s00330-0154011-X.

32. You C, et al. Association between background parenchymal enhancement and pathologic complete remission throughout the neoadjuvant chemotherapy in breast cancer patients. Transl Oncol. 2017;10(5):786-92. https://doi.org/10.1016/j.tranon.2017.07.005.

33. Oh SJ, Chae EY, Cha JH, Shin HJ, Choi WJ, Kim HH. Relationship between background parenchymal enhancement on breast MRI and pathological tumor response in breast cancer patients receiving neoadjuvant chemotherapy. Br J Radiol. 2018;91(1088):20170550. https://doi.org/10.1259/ bjr.20170550.

34. Leo GD, Trimboli RM, Sella T, Sardanelli F. Optical imaging of the breast: basic principles and clinical applications. Am J Roentgenol. 2017;209(1):2308. https://doi.org/10.2214/AJR.16.17220.

35. Noh JJ, Maskarinec G, Pagano I, Cheung LW-K, Stanczyk FZ. Mammographic densities and circulating hormones: a cross-sectional study in premenopausal women. Breast. 2006;15(1):20-8. https://doi.org/10.1016/j. breast.2005.04.014.

36. Bremnes Y, Ursin G, Bjurstam N, Rinaldi S, Kaaks R, Gram IT. Endogenous sex hormones, prolactin and mammographic density in postmenopausal Norwegian women. Int J Cancer. 2007;121(11):2506-11. https://doi.org/10. 1002/ijc.22971.

37. King V, Gu Y, Kaplan JB, Brooks JD, Pike MC, Morris EA. Impact of menopausal status on background parenchymal enhancement and fibroglandular tissue on breast MRI. Eur Radiol. 2012;22(12):2641-7. https:// doi.org/10.1007/s00330-012-2553-8.

38. K. D. Miller, C. J. Sweeney, and G. W. S. Jr, "Redefining the target: chemotherapeutics as antiangiogenics," J Clin Oncol. 2016, doi: https://doi. org/10.1200/JCO.2001.19.4.1195

39. Sala E, Warren R, McCann J, Duffy S, Luben R, Day N. High-risk mammographic parenchymal patterns and anthropometric measures: a case-control study. Br J Cancer. 1999;81(7):1257-61. https://doi.org/10.1038/ sj.bjc.6690838.

40. Stone J, Warren RML, Pinney E, Warwick J, Cuzick J. Determinants of percentage and area measures of mammographic density. Am J Epidemiol. 2009;170(12):1571-8. https://doi.org/10.1093/aje/kwp313.

41. Boyd NF, et al. Body size, mammographic density, and breast cancer risk Cancer Epidemiol Prev Biomark. 2006;15(11):2086-92. https://doi.org/10. 1158/1055-9965.EPI-06-0345.

42. Boyd NF, Lockwood GA, Byng JW, Little LE, Yaffe MJ, Tritchler DL. The relationship of anthropometric measures to radiological features of the breast in premenopausal women. Br J Cancer. 1998;78(9):1233-8.

\section{Publisher's Note}

Springer Nature remains neutral with regard to jurisdictional claims in published maps and institutional affiliations.

\section{Ready to submit your research? Choose BMC and benefit from}

- fast, convenient online submission

- thorough peer review by experienced researchers in your field

- rapid publication on acceptance

- support for research data, including large and complex data types

- gold Open Access which fosters wider collaboration and increased citations

- maximum visibility for your research: over $100 \mathrm{M}$ website views per year

At BMC, research is always in progress.

Learn more biomedcentral.com/submissions 